\title{
On Calibration Techniques for Pipelined ADCs
}

\author{
Swina Narula, and Sujata Pandey* \\ Amity School of Engineering \& Technology, Amity University, Noida \\ *Amity Institute of Telecom Engineering and Technology, Amity University Noida, INDIA \\ Email: spandey@amity.edu
}

\begin{abstract}
The development and designing of advanced pipelined analog to digital converter (ADC) is becoming sophisticated day by day as dimensions and supply voltages used for devices are reducing. As the nanometer technology aids fabricating circuits with small footprints, we require high-performance Pipelined ADCs, which are able to rectify analog circuit non-idealities with digital calibration circuits. Digital background calibration is the most favorable solution instead of making changes in analog components in the deep submicron processes. This paper discusses some of the techniques for digital calibration that have been accepted to realize advanced pipelined ADCs. It was observed that on an average, for every two years, the efficiency of ADCs is enhanced by a factor of two. With constant technology scaling supply voltages have reduced and the devices operate at high speed. A closer inspection on SNDR, SFDR, INL and DNL of pipelined ADCs with different calibration techniques is presented here. Finally, a comparison on various design approaches is made to make a view towards additional enhancement in speed, power efficiency and functioning of ADCs.
\end{abstract}

Key words: pipelined ADCs, calibration techniques, SNDR, SFDR, INL, DNL

\section{INTRODUCTION}

There are variety ofapplications of pipelined ADCs like broadband communication transceivers, video imaging systems, cable head ends i.e. for digitizing cable modem uplinks, medical instrumentation, professional HDTV cameras, cellular base station and military. The Pipelined ADC having multistage architecture deals with a solution for low power, high resolution, high speed and chip area. Applications based on low resolution requirement like 8-10 bit resolution may use various power reduction techniques [1-3]. It's really hard to achieve the speed of the ADCs exceeding $100 \mathrm{MS} / \mathrm{s}$ with resolution beyond 12-bit without applying any calibration technique. As the resolution of ADC increases, the hardware complexity also increases. Due to switched capacitor circuits used in MDAC of pipelined ADC, various errors like op amp nonlinearity, capacitor sizing error andOP-AMP with finite gain may come in picture.

As CMOS technologies are scaling down, amplifiers with high gain are difficult to fabricate. Also capacitor sizing issues degrades the performance of ADCs. There are so many techniques available to minimize these errors [4-10]. All these techniques have their own advantage, disadvantages and limitations. A comparison of these techniques for digital calibration is not available in literature till now.

The purpose of this paper is to analyze and compare different digital calibration techniques with their advantages that have been developed and used in various applications. Some important calibration techniques like nested background calibration, calibration using least mean square method, SPLIT, variable amplitude dithering, equalization based calibration, inter-stage scaling, two way interleaved and interpolation based nonlinear calibration has been discussed in details. There are five sections in this paper. Section II outlines pipelined ADCs with different errors and Section III emphasizes on the motivation behind the subject and different categories of calibration techniques. Section IV discusses the available techniques for digital calibration used for pipelined ADCs and their comparative analysis.

\section{PIPELINED ADC ARCHITECTURE AND DIFFERENT ERRORS}

In pipelined ADC's, $n$ number of individual ADC stages are cascaded [11-18]. SampleandHold (S/H) circuit, Sub-DAC (DigitaltoAnalog Converter), Sub-ADC, analog subtractor and again amplifier are working as building block for each stage [16-17].

The transfer function $\left(\mathrm{V}_{\text {res }}=2 \mathrm{~V}_{\text {in }}-\mathrm{V}_{\text {dac }}\right)$ of a pipelined $\mathrm{ADC}$ is usually implemented by Switched capacitor (SC) circuits in CMOS technology and Figure 1 shows the block diagram of a one stage of a pipelined ADC. 


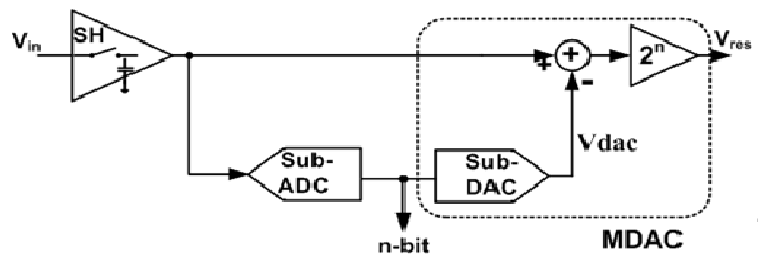

Figure 1: Block diagram of pipelined ADC stage

\section{Correction Logic}

At each clock cycle, the sample and hold block samples the input voltage $\left(\mathrm{V}_{\text {in }}\right)$ and holds the sampled value, to produce a low resolution digital output. The DAC converts the digital output back to analog $\left(\mathrm{V}_{\mathrm{dac}}\right)$ so that it would be subtracted from input voltage and produce the new output voltage known as residue of that particular stage. A 10 bit pipelined ADC which includes 1.5-bit/stage as a single stage architecture, is well available in literature. A $1.5 \mathrm{bit} / \mathrm{stage}$ architecture offers advantages such as stage is more immune to comparator offset as far as offset is within $\pm V_{r e f} / 4 . V_{r e f}$ is the reference voltage of ADC. This is accomplished with the help of Digital Error Correction Logic (DECL), consisting of adders, as shown in Figure 2 [16-17].

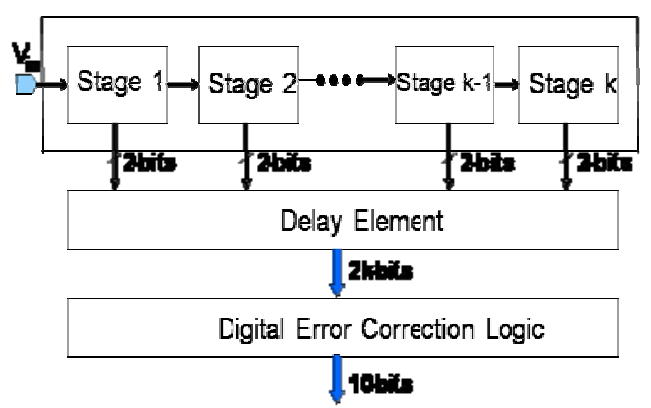

Figure 2: Complete Pipelined ADC with delay elements and DECL

In each stage, delay units have to be used to synchronize the digital outputs of different stages. For a 10 bit Pipelined ADC, there will be latency of 10 clock pulses [16-17].

\section{Different errors:}

Multiplying DAC (MDAC) is the cause of different errors: finite DC gain of op-amp, capacitor mismatch $\left(\mathrm{C}_{\mathrm{F}} / \mathrm{C}_{\mathrm{S}}\right)$ and comparator offset $\left(V_{\text {off }}\right)$.

\section{Capacitor mismatch error:}

The capacitor ratio $\mathrm{C}_{\mathrm{S}} / \mathrm{C}_{\mathrm{F}}$ is important for deciding the gain of a Switched Capacitor MDAC. Different values of the capacitors $\mathrm{C}_{\mathrm{S}}$ and $\mathrm{C}_{\mathrm{F}}$ may change the residue output. So, for better resolution pipelined ADC, an accurate capacitor matching is required [18-20]. Because of fabrication issues, over-etching and the oxide- thickness gradient may cause capacitor mismatch.The effect of capacitor mismatch error on residue and ADC output is shown in Figure 3.

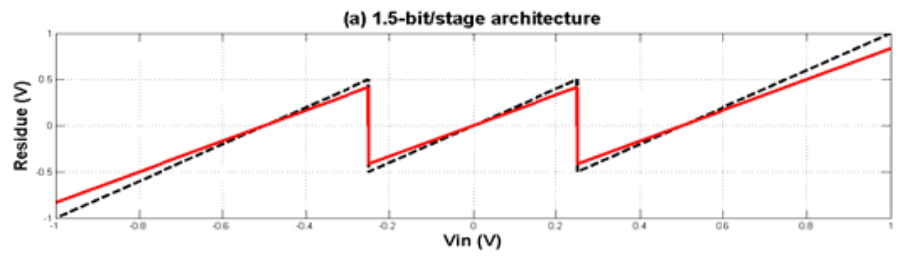

(b) ADC output

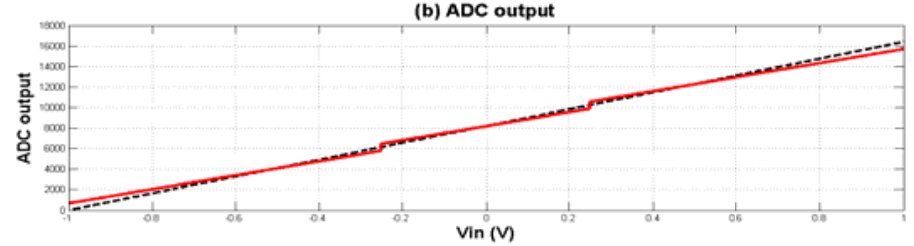

Figure 3: Capacitor mismatch error

The dotted line shows the graph of an ideal transfer function and the solid red line shows the output with capacitor mismatch error [16-17]. 


\section{Comparator offset:}

An internal offset voltage may add to the difference produced by a comparator.Thus, the output of the comparator propagates through other stages and wrong output is produced. The effect of comparator offset error on residue and ADC output as a function of input voltage is shown in Figure 4.The dotted line shows the desired output and the solid line shows the erroneous output[16-17].

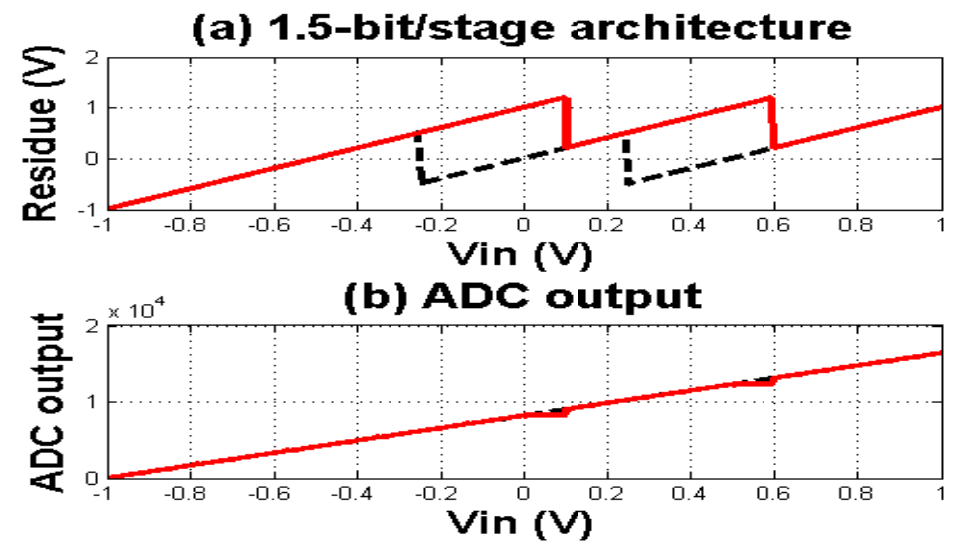

Figure 4: Comparator offset error

OP-AMP's finite open loop gain: Since an op-amp plays an important role in Switched capacitor circuit [6], it is essential to show the effect of non-idealities of op-amps. A Switched capacitor implementation is shown in Figure 5.

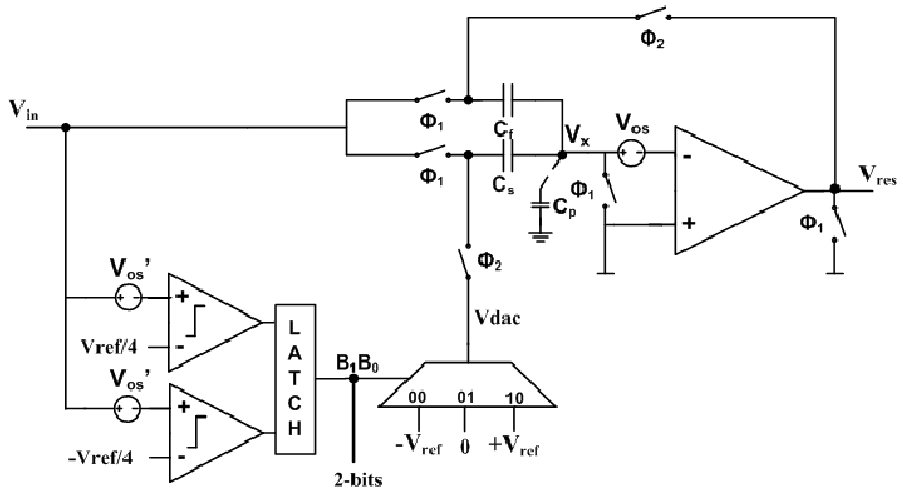

Figure 5: 1.5-bit per stage SC implementation

$\mathrm{C}_{\mathrm{P}}$ is the input parasitic capacitance and A is the open loop DC gain of the op-amp.

Motivation: Non-calibrated Pipeline ADCs can achieve less than 11 bits resolution and the calibrated pipeline ADCs resolution can be improved to more than 14 bits. The motivation for calibration is to improve both the static and dynamic behavior of ADCs which includes Offset error, full scale error, Gain error, Signal to Noise and Distortion Ratio (SNDR), Spurious Free Dynamic Range (SFDR), Effective Number of Bits (ENOB), Total Harmonic Distortion (THD), Differential Non Linearity error (DNL), Integral Non Linearity Errors (INL) etc. Different types of errors can be corrected through calibration. So, extra circuitry is required, which can monitor the ADC output, identify errors and rectify the output. Choice of calibration technique depends upon accuracy, speed and application of ADC. Different categories of calibration techniques are performed like foreground calibration and background calibration [5-7].

Factory Calibration: Using factory calibration, one-time events like capacitor trimming can be resolved perfectly. To improve the linearity of the ADC, trimming of capacitors is done to match the capacitors and this could happen before packaging the ADC, which can be achieved easily by factory calibration.

Foreground Calibration: Here re-calibration is allowed at power up but at the time of calibration it requires the converter to be off-line. The major drawback associated with this scheme for calibrating ADCs, the normal operation of $\mathrm{ADC}$ interrupts and hence requires extra clock cycles to inject calibration signal, which results in slow response [12]. But this scheme is non-transparent to all ADCs.

Continuous Calibration: The best part of continuous calibration is its ability to do calibration in the background Environmental without disturbing the normal operation of ADC i.e analog input signal is converting into digital output signal simultaneously. Further two types are available here one is Analog and other is Digital. 
Analog: The drawbacks of analog precision techniques are the extra cycles required for calibration, slow response and increased power dissipation. Moreover, the process is not transparent to all ADCs. As the down scaling continues in the process technologies, it is usually difficult to scale the analog calibration scheme.

Digital: This is the most popular type of calibration as the converter remains in its normal mode of operation while calibration is being processed. For deep submicron technologies, digital background calibration is the best solution. It is more robust and cheap for high-performance and complex processing. It's really quick to market with changes required as only digitally modifications. Accuracy and speed of pipelined ADCs is relaxed using digital correction techniques. Different techniques of digital calibration have been introduced for Pipelined $\mathrm{ADCs}$ in last few years e.g. LMS (Least-Mean Square method), using variable amplitude dithering, Split method etc.

\section{1) Nested Background Calibration}

\section{COMPARISON OF CALIBRATION TECHNIQUES}

In ADCs it's very hard to achieve speed and accuracy both together. Practically low speed ADCs are more accurate than high-speed ADCs. In the Nested Background Calibration scheme [6], the architecture used for $\mathrm{ADC}$ is accurate but slow, which is cautiously calibrating a $\mathrm{ADC}$ which is fast but inaccurate. The calibration is nested because slow-but-accurate ADC, which has been already calibrated in foreground, is used here as a reference $\mathrm{ADC}$ to calibrate inaccurate pipelined ADC. This calibration technique (nested background calibration) is based on LMS (Least mean square) algorithm [20]. Using digital signal processing unit, calibrated output generated [6] using this technique is shown in Figure 6.

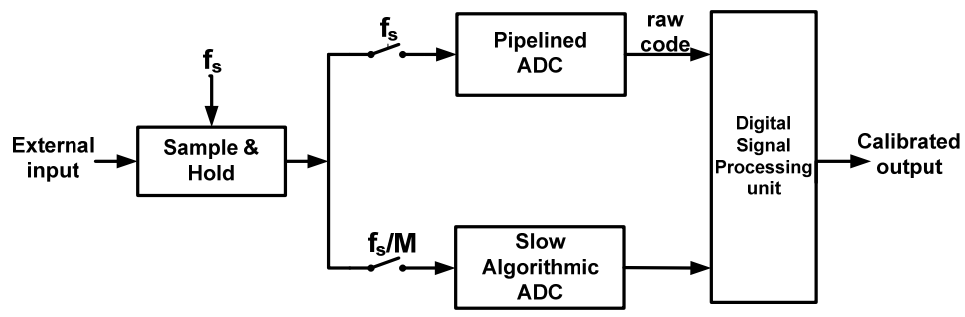

Fig 6: Nested back ground calibration [6]

At the same sampling rate of 20-Msample/s, both the sample and hold amplifier (SHA) and the Pipelined ADC operates. The gain error information, about the pipelined ADC, saves in the form of raw code and it have to be taken out in the digital error estimation block (DEE). To produce the calibrated output, the un-calibrated output is combined with output of DEE block. The erroris produced by subtraction of pipelined and algorithmic outputs. Adders in the DEE block and negative feedback in association is used to reduce the MSE, so the calibrated output lines optimize the output of the slow algorithmic ADC in steady state. The advantage of this scheme is that high gain op-amps are not required and circuit non idealities because of capacitor mismatch is also removed. Different problems may occur with this calibration scheme such as area overhead. The reference $\mathrm{ADC}$ may have a complicated circuit of the type folding interpolating architecture resulting in increased power consumption.

\section{2) Calibration using Least-Mean Square method}

By adjusting the analog component values to attain linearity, the new method assumes component errors from conversion results, which uses digital post processing on results to correct those errors. This scheme resembles the channel equalization problem, which occurs in digital communication system. Here, using a slow but accurate ADC, the code domain adaptive finite impulse response (FIR) filter is used to remove the effect of component errors [7]. This scheme is able to remove errors like op-amp offset error, capacitor mismatch; sampling switch induced offset and finite op-amp gain, which is not dependent on signal. The block diagram utilizing this algorithm, running in the background, which is digital, fully adaptive and data driven is shown in figure 7.

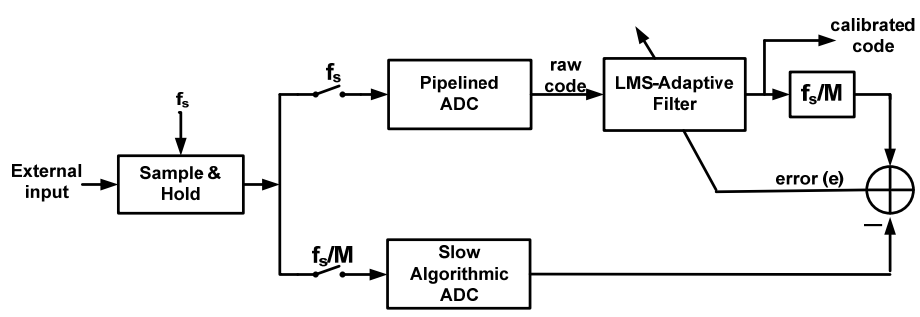

Fig 7: code-domain LMS adaptive LE as digital correction system 
The benefit of this scheme is relaxing from the requirement of precision of analog components and is able to continue CMOS device scaling approach [8]. Component errors from all stages are removed by Finite impulse response (FIR) digital filter. The analog signal path is intact completely to maintain conversion speed as maximum permitted by device technology used for designing purpose. This is able to correct errors caused by finite op-amp gain, capacitor mismatch, various input-referred offsets etc. A Pipelined ADC has been implemented in $0.35-\mu \mathrm{m}$ double-poly triple-metal CMOS process [21]. Also it is able to achieve 14-b accuracy without calibration or dithering but it is required to work at high input voltage and it dissipates large power.

A combination of techniques is used to improve accuracy, such as amplifiers with gain boosting, domainextended digital error corrections, communicated feedback capacitor switching (CFCS) and low noise dynamic comparators [22]. Also front-end stage uses a sample and hold amplifier (SHA) - less front end stage.

\section{3) Calibration using SPLIT}

In this technique, a single $\mathrm{ADC}$ is split into two $\mathrm{ADC}$, where individual converter is converting the same input signal. Two outputs from two ADCs is averaged to produce calibrated digital signal as shown in Figure 8[9].

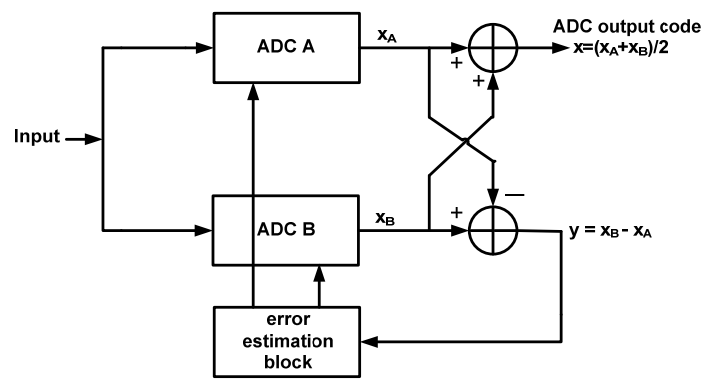

Fig 8: Split based ADC calibration technique [9]

The final ADC output is achieved by averaging the two different outputs from two different ADCs. The difference of outputs of both ADCs is equal to zero with the same input, indicates ADCs are well calibrated. The difference with non-zero value indicates calibration is required and the value decides the amount by which it should be calibrated. As analog circuit broke up into 2, so total analog area remains same. As we know bandwidth is proportional to $g m / C$, power is propostional to $g m$ and noise is proportional to $\sqrt{k T} / C$, splitting by two parts and having capacitance of $\mathrm{C} / 2$ by each half, bandwidth remains same and so power. The overall noise remains unchanged as the results are getting by averaging of two [9]. For a 10-bit, $1 \mathrm{MS} / \mathrm{s}$ algorithmic ADC, Self-calibration is used with around 10000 conversions. By using the concept of split ADC, the analog area of single ADC essentially splits into two, so it makes insignificant effect on analog complexity when consider its power, overall area, noise performance and bandwidth [23]. But the concept of split ADC only, is not enough for the estimation of errors. For example, if both A and B sides have the same error and comparators outputs also same, it would be unable to rectify the error [15]. But this problem could be rectified if we force the two sides to take different decisions and it can be realized by multiple residue mode cyclic amplifier [9]. This amplifier will be the combination of dual residue approach and the $1.5 \mathrm{bit} / \mathrm{stage}$ amplifier. Which comparator output will be used for digital output has been decided by 2 bit path address. The best possible residue among four residue modes, this technique helps for digital output selection without interacting the analog circuit. But it requires an additional comparator. It will impact minimal on overall area and power. Due to multiple residue mode, a wide variety of decision paths are available, so it is easy to extract the calibration information even for a DC input.

\section{4) Calibration using variable amplitude dithering}

To measure domain-extended Pipelined ADC gain errors, the pseudo-random noise dither (PN dither) method is majorly used for digital calibration. By using redundancy bits, the digital error correction technique has been applied to rectify the comparator offset error. But there are some disadvantages with these two techniques like decrease in amplitude of the transmitting, slow convergence speed and deduction of the redundancy space [2427]. To overcome these disadvantages, instead of using the pseudorandom noise dither, the variable-amplitude dithering has been used for a digital calibration algorithm for domain-extended pipelined ADCs [10]. This circuit works well even in worst conditions like the signal stays at high level all the time. The redundancy space plus the total amplitude of the signal and the dither has been restricted by the quantify range. Both static and dynamic performances have improved after the calibration with much higher convergence speed without any circuit complexity. The amplitude of the dither varies with the signal level. Because the amplitude of the dither can be increased without any loss of the amplitude of the signal, the convergence speed remains high. Further over, in the domain-extended architecture, due to the existence of more redundancy space it allows the comparator offsets to be corrected that is within a certain range. 
In this case, the values of threshold voltages have changed to $\pm \frac{3}{4} V_{r e f}, \pm \frac{1}{4} V_{r e f}$, where the input range is from $\frac{5}{4} V_{\text {ref }}$ to $\frac{5}{4} V_{\text {ref }}$ which is same as output range. Here the the value of $V_{\text {ref }}$ is equal to half of the power supply voltage $V_{d d}$. With the variable-amplitude dithering, three more comparators are added and a capacitor is spilled into four capacitors, $C_{1}, C_{2}, C_{3}$ and $C_{4}$ with each capacitor valued at $\frac{C_{f}}{4}$. Switches have used to control dither injections which depends on both the output of the encoder and the PN value. The amplitudes of the dithers from $-\frac{3}{4} V_{\text {ref }}$ to $-\frac{1}{4} V_{\text {ref }}$ in all sub-levels of the main level, is shown in Table 1 .

Table 1: Amplitude of the dithers

\begin{tabular}{|c|c|c|c|}
\hline \multicolumn{2}{|c|}{$V_{\text {in }}$} & \multicolumn{2}{|c|}{ Amplitude of dithers } \\
\hline Main level & Sub-levels & $P N=-1$ & $\mathbf{P N}=1$ \\
\hline$-\frac{3}{4} V_{r e f} \sim-\frac{1}{4} V_{r e f}$ & $-\frac{3}{4} V_{r e f} \sim-\frac{5}{4} V_{r e f}$ & $\frac{5}{4} V_{\text {ref }}$ & $-\frac{1}{2} V_{r e f}$ \\
\hline & $-\frac{5}{8} V_{r e f} \sim-\frac{1}{2} V_{r e f}$ & $V_{\text {ref }}$ & $-\frac{3}{4} V_{r e f}$ \\
\hline & $-\frac{1}{2} V_{r e f} \sim-\frac{3}{8} V_{r e f}$ & $\frac{3}{4} V_{\text {ref }}$ & $-V_{\text {ref }}$ \\
\hline & $-\frac{3}{8} V_{r e f} \sim-\frac{1}{4} V_{r e f}$ & $\frac{1}{2} V_{r e f}$ & $-\frac{5}{4} V_{r e f}$ \\
\hline
\end{tabular}

\section{5) Equalization based digital calibration}

In equalization based digital calibration technique, the calibration signals are normally used to measure the errors. But, in the deep submicron technologies, the precision of these signals is very important. Various techniques are available [32-34].

The amplifier nonlinearity, capacitors mismatch and residue gain error are measured first and corrected later in digital domain by using Equalization based digital calibration. This scheme uses both foreground and background calibration methods. In foreground, the error estimation has done with non-precision calibration signals and to convert foreground to background scheme, and an adaptive linear prediction structure has been used. Here, the LMS algorithm has been used as the foreground technique to estimate the error coefficients which didn't need high accuracy signals for calibration. Here CNFA MDAC topology has been used to design the 1.5-bit/stage pipelined ADC [11]. The process of calibration works recessively in opposite direction by pipelined stages as shown in Figure 9.

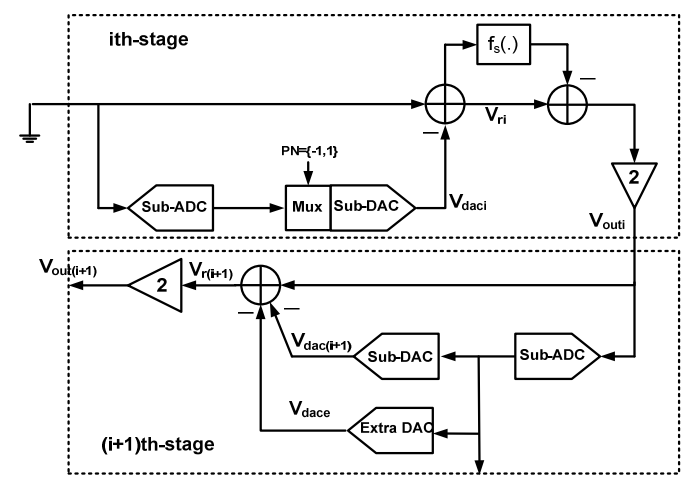

Fig 9: Calibration of ith stage implemented in CNFA MDAC structure [11].

This technique for Pipelined ADCs, does not require any accurate calibration signal. The adaptive LMS algorithm [8] has been used to approximate the errors.

\section{6) Inter stage scalingfor low power Pipelined ADC}

Here, implementation of a pipelined ADC has been done in deep submicron technology e.g. $0.13 \mu \mathrm{m} \mathrm{CMOS}$ technology. In [12], the ADC is made up of a Sample and Hold circuit( $\mathrm{S} / \mathrm{H})$, eight cascading 1.5-bit stage and a 2-bit Flash ADC. The block of current generator, non-overlapping clock, reference generator and Digital Error Logic (DEL) is also required to correct the offset of the comparator and is shown in Figure 10. 


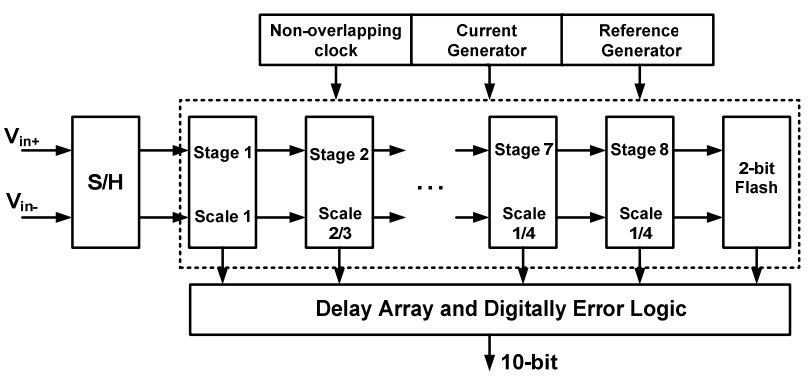

Fig 10: Block diagram of Pipelined ADC [12]

The bootstrapped switch in the SHA, is used to minimize the distortion. Switched-capacitor comparator is used in Sub-ADC. MDAC uses the $2 \mathrm{X}$ gain in each stage. The first stage has been designed with specific considerations to noise, distortion and incomplete settling. Moreover, high bias currents and large sampling capacitors are used. For the second stage, the downscaling factor is $2 / 3$ and $1 / 4$ for the further stages. OTA is specifically designed to achieve the sampling rate of $50 \mathrm{MHz}$ with $1.6 \mathrm{pF}$ load to minimize the settling time. As the scale factor is $2 / 3$ in the stage 2 , the sampling capacitor $(\mathrm{C} 1)$ and the feedback capacitor $(\mathrm{C} 2)$ can be reduced to about $2 / 3$ as compared to $\mathrm{C} 1$ and $\mathrm{C} 2$ in the stage1. In [28], the multi-bit architecture has been used with a very less op-amp count that is three only for the complete ADC rather than using a count of eight as in a standard 1.5 bit Pipelined ADC architecture. An inter-stage scaling has been applied very effectively to all stages of Pipelined ADC by using the same unary cell implementation and also the cell has been used multiple at higher stages. Along with the pipeline stages, the power consumption and area consumption decreases without compromise with the performance of this converter with the downscaling of technology also. The problem with this calibration scheme is sampling frequency can't go high as required in some applications. There are limitations to increase the resolution of Pipelined ADC, which is required for many applications. In some cases the system works a little bit slow. In [29], the 1.5-b/stage Pipelined ADC core has been implemented using an amplifier sharing technique, capacitor matching layout and swing-improved telescopic OTA. The ADC has been designed specially to work at high input voltage and least power dissipation with excellent static and dynamic performance.

\section{7) Two-way time-interleaved Pipelined ADC occupying less Area}

With this adaptive power/ground architecture eradicates the headroom boundaries due to low power requirement. While considering the signal swing, this technique gives a flat transition between the MDAC stages and the last flash stage. A single-stage amplifier structure has been used in the SHA and MDACs to attain good phase margin, wide bandwidth and low noise is chosen. A telescopic cascade structure has been used to achieve high amplifier gain to get 12-bit linearity. To improve the bandwidth, all input devices are implemented with thin gate device only of a gain-boosting circuit. For CMOS Switch, in the amplifier design, a $2.5 \mathrm{~V}$ supply is used. The disadvantage of thick-oxide devices is to make it slow and normally thin-oxide devices are more reliable in terms of fast transition, low on-resistance and very less power consumption. A 6-level Flash ADC is used to generate a 2.5-bit digital output in each MDAC and the final residue is digitized by a 6 bit Flash ADC. In [30], to generate the extra reference voltage taps, a reference voltage extrapolation method is used here. From the inner taps of the reference ladder, three-input dummy pre-amplifiers at the edges generate the over ranging voltages by analog addition. In both 6-bit Flash ADC and the 2.5-bit Flash ADCs, MDACs and the folded preamplifiers are used. In [31], an 11-bit time-interleaved ADC with 800MS/S has been implemented for a 10 GBase-T application in a $90 \mathrm{~nm}$ CMOS process. To achieve high resolution and conversion rate, a single open-loop T/H circuit using a cascade source follower has been used.

\section{Interpolation based non - linear calibration}

In high resolution pipelined ADCs, the utmost requirement is high gain OP-AMP and large capacitors. But these are the main cause of large ADC power. The linearity of ADC is also limited by the finite operational amplifier (OPAMP) gain and capacitor mismatch in MDAC. In [14], interpolationbased digital self-calibration architecture for pipelined $\mathrm{ADC}$ has been introduced. The $0.78 \mathrm{pJ} / \mathrm{step}$ figure-of-merit (FOM) is low for designs in $0.35 \mu \mathrm{m}$ CMOS processes. Here a precise Cal ADC is used to measure the MDAC error and it runs in two modes as calibration mode and recovery mode.

Calibration Mode: It enables Cal ADC to measure any internal node in the pipeline, so that MDAC transfer function can be measured directly. A set of input voltages is supplied to the pipeline ADC. CalADC switches are used to measure the MDAC transfer function. Switches will turn on successively, while complimentary switches turn off at the same time. Every time, every MDAC on which calibration is going on, a sample on its transfer function is recorded every time $(\mathrm{X} \mathrm{j}, \mathrm{i}, \mathrm{Y} \mathrm{j}, \mathrm{i})$, where $\mathrm{X} \mathrm{j}, \mathrm{i}$ is the input voltage of the $\mathrm{i}$-th sample and $\mathrm{Yj}, \mathrm{i}$ is the output voltage of the $\mathrm{i}$-th sample. 
Recovery Mode: In this mode, all switches goes off and Cal ADC is held away from the pipelined ADC. The 14-bit raw codes are digitized from input signal by pipeline $\mathrm{ADC}$, converted into 12 bit code by a digital decoder. In this design, the pipeline is to generate 13-bit raw data and the extra 1-bit is for design redundancy. From the end of the pipeline, the recovery process is started and moves in order to the front in this process, MDAC input voltage is calculated using the residue voltage and its quantization code. At the end of the pipeline, for MDACs, there is no requirement of calibration, as they considered linear. As this architecture is not using backend ADC to measure MDACs, it is free from the measurement error, which results in more accurate calibration. Because of the slow settling of the first pipeline stage, SNDR of the calibrated ADC drips to $8 \mathrm{~dB}$ at the Nyquist frequency. Also the power consumption is high.

Table 2 shows, all the performance parameters of different calibration techniques, which are most popular for Pipelined ADCs.

Table 2: Comparison of the performance of Pipelined ADCs having different calibration techniques

\begin{tabular}{|c|c|c|c|c|c|c|c|c|c|c|c|}
\hline $\begin{array}{l}\text { Ref } \\
\text { ere } \\
\text { nce } \\
\text { No. }\end{array}$ & $\begin{array}{l}\text { Res } \\
\text { olut } \\
\text { ion } \\
\text { (bit } \\
\text { ) }\end{array}$ & $\begin{array}{l}\text { VDD } \\
(\mathrm{V})\end{array}$ & $\begin{array}{l}\text { Area } \\
\text { used } \\
(\mathrm{mm} 2\end{array}$ & $\begin{array}{l}\text { Tec } \\
\text { hnol } \\
\text { ogy } \\
\text { used }\end{array}$ & $\begin{array}{l}\text { THD } \\
(\mathrm{dB})\end{array}$ & $\begin{array}{l}\text { Sampling } \\
\text { freq }\end{array}$ & $\begin{array}{l}\text { SND } \\
\text { R } \\
(\mathrm{dB})\end{array}$ & $\begin{array}{l}\text { SFD } \\
\text { R(d } \\
B)\end{array}$ & $\begin{array}{l}\text { INL( } \\
\text { LSB) }\end{array}$ & $\begin{array}{l}\text { DNL( } \\
\text { LSB) }\end{array}$ & $\begin{array}{l}\text { Power } \\
\text { diss.(mw) }\end{array}$ \\
\hline [6] & 12 & 3.3 & 7.5 & $\begin{array}{l}0.35 \\
\mu \mathrm{m} \\
2 \mathrm{P} 4 \\
\mathrm{M}\end{array}$ & -92.9 & $20 \mathrm{MS} / \mathrm{s}$ & 70.8 & 93.3 & $\begin{array}{l}0.47 \\
(\max )\end{array}$ & $\begin{array}{l}0.41( \\
\max )\end{array}$ & 254 \\
\hline [8] & 12 & 3 & 7.9 & $\begin{array}{l}0.35 \\
\mu \mathrm{m}\end{array}$ & $\begin{array}{l}76(1 \\
\mathrm{mHz}) \\
74(40 \\
\mathrm{MHz})\end{array}$ & $75 \mathrm{MS} / \mathrm{s}$ & - & $\begin{array}{l}80(1 \\
\mathrm{MHz} \\
) \\
76( \\
40 \\
\mathrm{MHz} \\
)\end{array}$ & $\begin{array}{l}- \\
0.9,+ \\
0.6\end{array}$ & $\begin{array}{l}- \\
0.5,+ \\
0.5\end{array}$ & 290 \\
\hline [9] & 16 & 2.5 & $\begin{array}{l}1.16 \\
\mathrm{mmX} \\
1.38 \\
\mathrm{~mm} \\
\end{array}$ & $\begin{array}{l}0.25 \\
\mu \mathrm{m} \\
1 \mathrm{P} 4 \\
\mathrm{M} \\
\end{array}$ & & $1 \mathrm{MS} / \mathrm{s}$ & - & - & $\begin{array}{l}+2.1 /- \\
4.8\end{array}$ & $\begin{array}{l}+0.66 \\
/-0.47\end{array}$ & 105 \\
\hline $\begin{array}{l}{[10} \\
]\end{array}$ & 12 & - & - & - & 86.2 & $100 \mathrm{MS} / \mathrm{s}$ & 87.7 & - & $\begin{array}{l}- \\
0.5 \sim 0 \\
.4\end{array}$ & $\begin{array}{l}- \\
0.4 \sim 0 \\
.5 \\
\end{array}$ & V. high \\
\hline $\begin{array}{l}{[11} \\
]\end{array}$ & 12 & - & - & $\begin{array}{l}90 \\
\mathrm{~nm}\end{array}$ & - & $100 \mathrm{MS} / \mathrm{s}$ & 68 & 83 & 2.2 & 0.9 & 48 \\
\hline $\begin{array}{l}{[12} \\
]\end{array}$ & 10 & 2.5 & 0.6 & $\begin{array}{l}0.3 \mu \\
\mathrm{m}\end{array}$ & - & $50 \mathrm{MHz}$ & - & 68 & $\begin{array}{l}- \\
0.63 / \\
+0.63 \\
\end{array}$ & $\begin{array}{l}- \\
0.27 / \\
+0.21 \\
\end{array}$ & 50 \\
\hline $\begin{array}{l}{[13} \\
]\end{array}$ & 12 & 2.5 & 0.4 & $\begin{array}{l}40 \\
\mathrm{~nm}\end{array}$ & & $3 \mathrm{GS} / \mathrm{s}$ & 58 & - & $\begin{array}{l}+03 /- \\
03\end{array}$ & $\begin{array}{l}+0.5 /- \\
0.5 \\
\end{array}$ & 500 \\
\hline $\begin{array}{l}{[14} \\
]\end{array}$ & 12 & 3.3 & $\begin{array}{l}4.8 \mathrm{~m} \\
\mathrm{mX} \\
4.3 \\
\mathrm{~mm} \\
\end{array}$ & $\begin{array}{l}0.35 \\
\mu \mathrm{m}\end{array}$ & -79.8 & $20 \mathrm{MS} / \mathrm{s}$ & 72.5 & 84.4 & 0.2 & 0.27 & 56.3 \\
\hline $\begin{array}{l}{[32} \\
]\end{array}$ & 12 & 2.5 & - & $\begin{array}{l}0.25 \\
\mu \mathrm{m} \\
\end{array}$ & - & $80 \mathrm{MS} / \mathrm{s}$ & 72.6 & 84.5 & +0.24 & +0.09 & 340 \\
\hline $\begin{array}{l}{[33} \\
] \\
\end{array}$ & 12 & 1.2 & - & $\begin{array}{l}90 \\
\mathrm{~nm}\end{array}$ & - & $200 \mathrm{MS} / \mathrm{s}$ & 62 & - & +1.3 & +0.59 & 348 \\
\hline $\begin{array}{l}{[34} \\
]\end{array}$ & 10 & 1.2 & - & $\begin{array}{l}90 \\
\mathrm{~nm}\end{array}$ & - & $500 \mathrm{MS} / \mathrm{s}$ & 56 & - & 1 & 0.4 & 55 \\
\hline
\end{tabular}

While the technology is scaling down, analog designing of ADCs is a pretty challenging job. So, the digital calibration techniques for pipelined ADCs are the utmost need of the system to fulfil requirements like highspeed, low-power, quick response time, and rejection of noise signal and high-resolution. Figure 11 shows the trend of static performance of some Pipelined ADCs with different resolutions. 


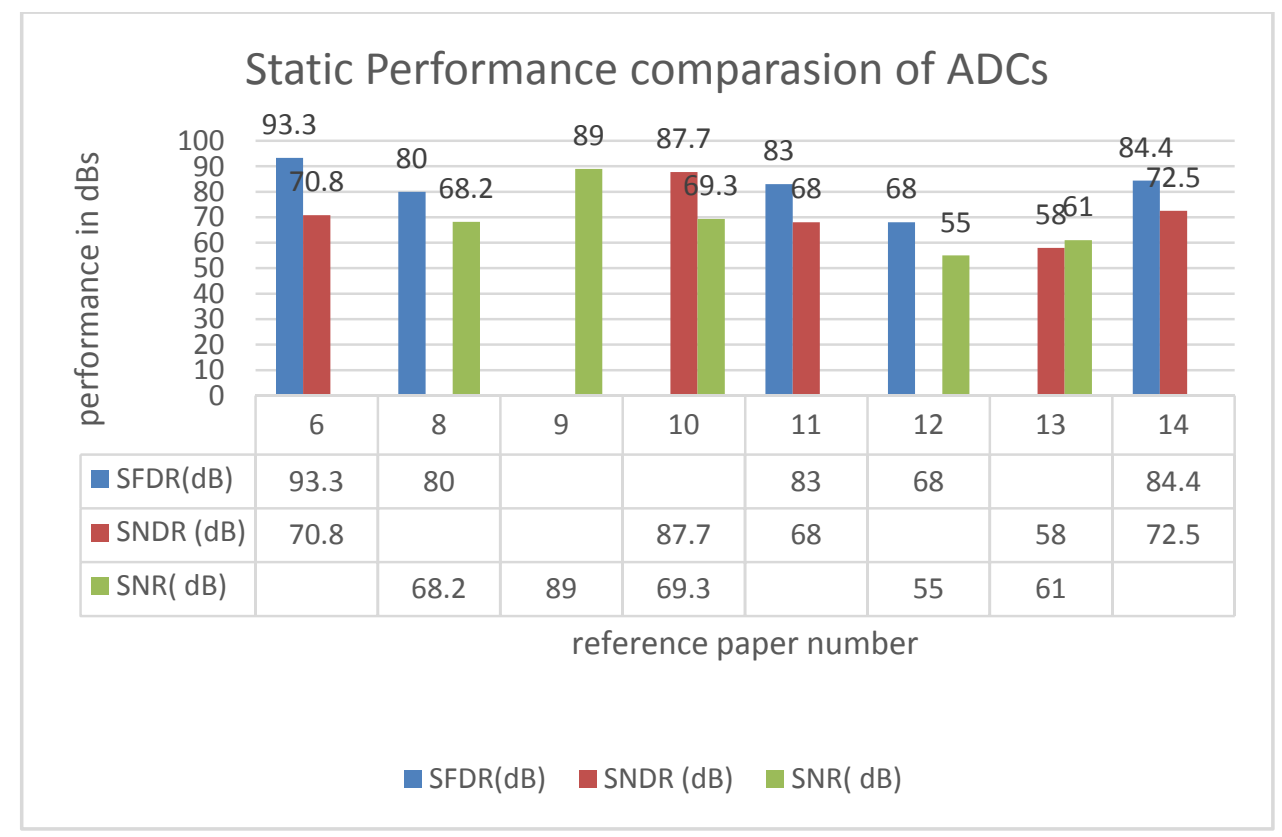

Figure 11: comparison of Static performance of different calibration techniques

We have observed from the papers that power efficiency in ADCs got upgraded at an amazing rate of $2 \mathrm{x}$ every 2 years using smaller feature sizes as shown in Figure 12.

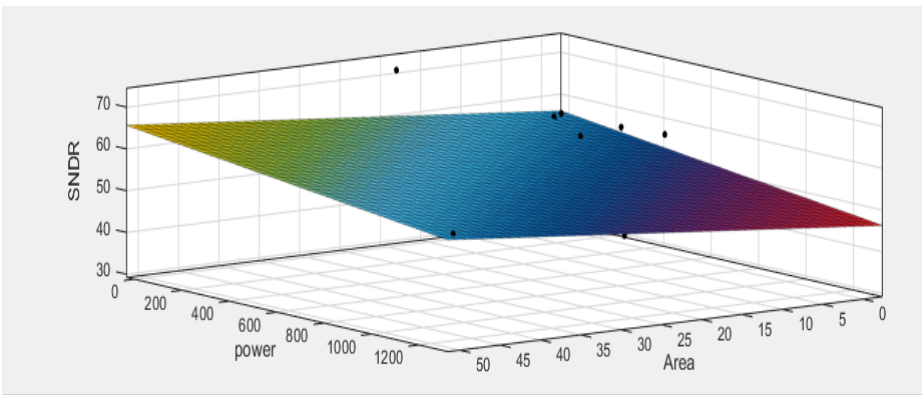

Figure 12: Trend of power dissipation and SNDR with area

Figure 13 shows the trend of power and SNDR increase with increase in area in last few years.

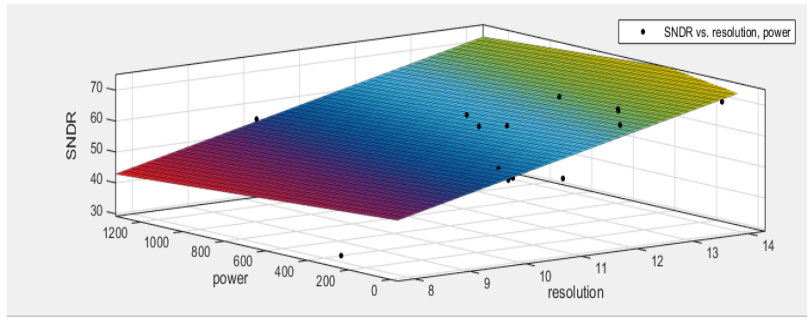

Figure 13: trend in power dissipation with resolution and SNDR

\section{CONCLUSION}

This paper discusses the requirement of digital calibration techniques developed for pipelined ADCs in the past few years and summarized different advantages and disadvantages, which may appear during operation. A favorable model is the drift towards smaller ADC architectures and digital circuits are used for error correction. Using digitally assisted ADCs aims to control the requirement of low power dissipation of modern processes and to enhance the resolution and static/dynamic performance of pipelined ADC circuits. In general, we can say that some improvements are still awaited to be a combination of features that involve improved system implanting and reducing analog sub-circuit complexity and a précise system with cheap digital processing resources. 


\section{References:}

[1] A. Panigada and I. Galton, "Digital background correction of harmonic distortion in pipelined ADCs," IEEE Trans. Circuits Syst. I, Reg. Papers, vol. 53, no. 9, pp. 1885-1895, Sep. 2006.

[2] A.Gharbiya, T.C. Caldwell, and D.A. Johns, "High speed oversampling analog-to-digitalconverters," International journal of high speed Electronics and systems, Vol. 15, No. 2, pp. 297-317, 2005

[3] N. Sasidhar, Y. Kook, S. Takeuchi, K. Hamashita, K. Takasuka, P. Hanumolu and U. Moon "A 1.8 V 36-mW 11-bit 80 MS/s pipelined ADC using capacitor and opamp sharing", IEEE Asian Solid-State Circuits Conf. 2007, ASSCC'07, pp.240 -243 2007.

[4] Wu, P.Y., Cheung, V.S.-L.; Luong, H.C. "A 1-V 100-MS/s 8-bit CMOS Switched-Opamp Pipelined ADC Using Loading-Free Architecture" IEEE Journal of Solid-State Circuits, Volume 42, Issue 4, April 2007.

[5] Li, J. ; Un-Ku Moon "Background calibration techniques for multistage pipelined ADCs with digital redundancy" IEEE Transactions on Circuits and Systems II: Analog and Digital Signal Processing, Volume:50, Issue: 9 , Sep 2003

[6] X.Wang,P.J.Hurst, S.H.Lewis, "A 12-bit 20-Msample/s Pipelined Analog-to-Digital Converter with Nested Digital background Calibration", IEEE Journal of Solid-State Circuits, vol.39, No.11, Nov. 2004.

[7] Yun Chiu, Cheong yuen W. Tsang, Borivoje Nikolic and Paul R. Gray, "Least Mean Square Adaptive Digital Background Calibration of Pipelined Analog-to-Digital Converters "IEEE Transactions On Circuits And Systems—I Vol. 51, No. 1, January 2004.

[8] Boris Murmann, Student Member, IEEE, and Bernhard E. Boser, Fellow, "A 12-bit 75-MS/s Pipelined ADC Using Open-Loop Residue Amplification" IEEE Journal of Solid-State Circuits, Vol. 38, No. 12, December 2003.

[9] John McNeill, Michael C. W. Coln and Brian J. Larivee,"Split ADC" Architecture for Deterministic Digital Background Calibration of a 16-bit 1-MS/s ADC "IEEE Journal Of Solid-State Circuits, Vol. 40, No. 12, December 2005

[10] Ting Li and Chao You "A Digital Calibration Algorithm With Variable-Amplitude Dithering For Domain-Extended Pipeline Adcs" International Journal of VLSI design \& Communication Systems (VLSICS) Vol.5, No.1, February 2014

[11] BehzadZeinali, TohidMoosazadeh, Mohammad Yavari and Angel Rodriguez-Vazquez, "Equalization-Based Digital Background Calibration Technique for Pipelined ADCs "IEEE Transactions On Very Large Scale Integration (VLSI) Systems, Vol. 22, No. 2, February 2014

[12] Zhzo-Xin Xiong and Min Cai "A 10-Bit 50-Ms/S Low-Power Pipeline ADC For Wimax/Lte" Journal of Theoretical and Applied Information Technology, May 2013. Vol. 51 No.3

[13] Chun-Ying Chen, Jiangfeng Wu, Juo-Jung Hung, Tianwei Li, Wenbo Liu and Wei-Ta Shih "A 12-Bit 3 GS/s Pipeline ADC With 0.4 $\mathrm{mm}$ and $500 \mathrm{~mW}$ in $40 \mathrm{~nm}$ Digital CMOS" IEEE JOURNALOFSOLID-STATECIRCUITS, VOL.47, NO.4, APRIL 2012

[14] JieYuan,Sheung Wai Fung, Kai Yin Chan and Ruoyu Xu "A 12-bit 20 MS/s 56.3 mW Pipelined ADC With Interpolation-Based Nonlinear Calibration" IEEE Transactions On Circuits And Systems-I: Regular Papers, Vol. 59, No. 3, March 2012

[15] John A. McNeill, "Digital Background-Calibration Algorithm for "Split ADC" Architecture", IEEE Transactions On Circuits And Systems-I: Regular Papers, Vol. 56, No. 2, February 2009.

[16] SwinaNarula, Sujata Pandey " A Methodology for Behavioral modeling of 10 bit Pipelined ADCs with modified Digital Error Correction Logic" Fifth international Conference on Advanced Computing and Communication Technologies, Jan 2015

[17] SwinaNarula, Sujata Pandey "High Performance 14-Bit Pipelined RSD ADC." inJournal of Semiconductors (IOP Science), Vol. 37, No.3, pp. , 2016.

[18] Moon, Un-Ku. Song, Bang-Sup. "Background digital calibration techniques for pipelined ADCs", IEEE Transactions on Circuits and Systems II- Vol. 44, No. 2, Feb 1997, pp 102.

[19] Gustavsson, M., J. J. Wikner, and N. N. Tan, "CMOS Data Converters for Communications" Kluwer Academic Publishers, 2000.

[20] X. Wang, P. J. Hurst, and S. H. Lewis, "A 12-bit 20-MS/s pipelined ADC with nested digital background calibration," in Proc. IEEE Custom Integrated Circuits Conf., Sept. 2003, pp. 409-412.

[21] W. Yang, D. Kelly, I. Mehr, M. T. Sayuk, and L. Singer, "A 3-V 340-mW 14-b 75-Msample/s CMOS ADC with 85-dB SFDR at Nyquist input,” IEEE J. Solid-State Circuits, vol. 36, pp. 1931-1936, Dec. 2001.

[22] Ting Li, Chao You "A 3-V 14-bit 75-MS/s CMOS pipeline analog-to-digital converter with 93.72-dB spurious-free dynamic range" International Journal of Electrical, Electronics and Computer Systems, March 2014.

[23] J. McNeill, M. Coln, and B. Larivee, “'Split-ADC" architecture for deterministic digital background calibration of a 16b 1MS/s ADC," in IEEE Int. Solid-State Circuits Conf. (ISSCC) Dig. Tech. Papers, Feb. 2005, pp. 276-278.

[24] E. Siragusa and I. Galton, "Gain error correction technique for pipelined analog-to-digital converters," Electron. Letter, vol. 36, pp. 617-618, Mar. 2000.

[25] E. Siragursa and I. Galton, "A digitally enhanced 1.8-V 15-bit 40-MSample/s CMOS pipelined ADC," IEEE Journal of Solid-State Circuits, vol. 39, no. 12, pp. 2126-2138, Dec. 2004.

[26] A. Panigada, I. Galton. "Digital background correction of harmonic distortion in pipelined ADCs," IEEE Trans. on Circuits and Systems I: Regular Papers, vol. 53, pp. 1885-1895, 2006.

[27] A. Meruva, and B. Jalali, "Digital background calibration of higher order nonlinearities in pipelined ADCs," IEEE International Symposium on Circuits and Systems, pp. 1233-1236, 2007.

[28] P. Bogner, "A 28mW 10b 80MS/s Pipelined ADC in $0.13 \mu \mathrm{m}$ CMOS", Proc. of the ISCAS 2004 Conf., pp. 17-20, 2004.

[29] Nan Wang, Ping Zhou, "A 10-bit 100-MS/s CMOS IP With Emphasis on layout Matching", IEEE Asian Conference of Solid-State Circuits, vol. 12, pp.335-358, Nov 2006.

[30] S.Gupta, M.Choi, M.Inerfield, and J.Wang, “A 1GS/s11b time-interleaved ADC in 0.13/spl mu/m CMOS," IEEE ISSCC Dig.Tech.Papers, 2006, pp. 2360-2369

[31] C.-C.Hsu, F.C.Huang, C.Y.Shih, C.C.Huang, Y.H.Lin, C.C.Lee, and B. Razavi, "An 11b 800 MS/s time-interleaved ADC with digital back ground calibration," in IEEE ISSCC Dig. Tech. Papers, 2007, pp. 464-465.

[32] C. R. Grace, P. J. Hurst, and S. H. Lewis, "A 12-bit 80-MSample/s pipelined ADC with bootstrapped digital calibration," IEEE J. SolidState Circuits, vol. 40, no. 5, pp. 1038-1046, May 2005.

[33] B. D. Sahoo and B. Razavi, "A 12-bit 200-MHz CMOS ADC," IEEE J. Solid-State Circuits, vol. 44, no. 9, pp. 2366-2380, Sep. 2009.

[34] A. Verma and B. Razavi, "A 10-bit 500-MS/s 55-mW CMOS ADC," IEEE J. Solid-State Circuits, vol. 44, no. 11, pp. 3039-3050, Nov. 2009. 